\title{
FATORES QUE GOVERNAM A ESPECIAÇÃO DO COBRE EM AMBIENTES AQUÁTICOS URBANOS: EVIDÊNCIAS DA CONTRIBUIÇÃO DE SULFETOS SOLÚVEIS
}

\author{
Elizabeth W. Scheffer, Fernando F. Sodré e Marco T. Grassi* \\ Departamento de Química, Universidade Federal do Paraná, CP 19081, 81531-990 Curitiba - PR, Brasil
}

Recebido em 3/1/06; aceito em 23/6/06; publicado na web em 19/1/07

\begin{abstract}
FACTORS GOVERNING COPPER SPECIATION IN URBAN FRESHWATERS: EVIDENCES ON THE CONTRIBUTION OF SOLUBLE SULFIDES. Copper speciation and behavior in different rivers located in the city of Curitiba were evaluated in this work. Sampling locations were selected to cover different levels of urbanization regarding their anthropogenic occupation and land use. Results showed that in highly-developed areas, both organic matter and dissolved sulfides were able to control copper speciation. Dissolved sulfide species were the major complexing agent in areas where dissolved oxygen levels are low. Finally, it was demonstrated that in urban areas anthropogenic factors such as sewage inputs and occupation of the drainage basin are the key aspects controlling copper dynamics and speciation in river waters.
\end{abstract}

Keywords: sulfide; copper speciation; natural waters.

\section{INTRODUÇÃO}

A especiação de metais em águas naturais consiste em aspecto chave no subsídio da previsão do seu comportamento geoquímico e de sua biodisponibilidade. Conseqüentemente, fornece informações importantes para avaliação do real impacto associado à presença dessas espécies em ambientes aquáticos. Em sua maioria, as análises de especiação de metais em águas naturais buscam um maior entendimento sobre o comportamento destas espécies, identificando e freqüentemente quantificando os agentes que governam a distribuição de metais na coluna de água e, conseqüentemente, sua biodisponibilidade.

No caso específico do cobre, sua especiação em águas naturais é influenciada por fenômenos físico-químicos, tais como a complexação por ligantes orgânicos e inorgânicos, a adsorção por oxi-hidróxidos metálicos e argilominerais e as reações de troca que ocorrem na interface água-sedimento ${ }^{1-5}$. A interação do cobre com a matéria orgânica dissolvida ou com o material particulado em suspensão, por ex., levam à formação de espécies ditas não biodisponíveis do metal ${ }^{4-6}$.

Muitos trabalhos mostram que a complexação do cobre pela matéria orgânica dissolvida pode governar tanto sua especiação ${ }^{7-12}$ quanto sua solubilidade ${ }^{13}$ em águas superficiais. Mais recentemente, entretanto, algumas pesquisas têm evidenciado que os sulfetos solúveis podem desempenhar um papel tão importante na especiação do cobre quanto o da matéria orgânica dissolvida, sendo que a disponibilidade deste metal para a biota pode ser governada por ambos os tipos de ligantes ${ }^{14-16}$.

As primeiras evidências da importância dos sulfetos solúveis na especiação de metais surgiram a partir do final dos anos $80 \mathrm{em}$ pesquisas realizadas em ambientes marinhos ${ }^{17-19}$, onde foi constatada a presença de $\mathrm{H}_{2} \mathrm{~S}$ em águas marinhas ricas em oxigênio e concentrações de $\mathrm{S}^{2-}$ que variavam entre 0,1 e $1,1 \mathrm{nmol} \mathrm{L} \mathrm{L}^{-1}$. Estas pesquisas também mostraram que existe uma relação entre a presença dos sulfetos e o controle da biodisponibilidade de metais, por meio da formação de complexos metálicos estáveis.

A existência de sulfetos em águas óxicas tem sido atribuída à elevada estabilidade dos complexos formados com espécies metá-

*e-mail:mtgrassi@quimica.ufpr.br licas $^{20,21}$. De forma que, os complexos metálicos de sulfeto se mostram essenciais tanto para a estabilização do sulfeto de hidrogênio em águas ricas em oxigênio, quanto para o controle da solubilidade de metais-traço.

No que diz respeito a águas continentais contendo teores de oxigênio dissolvido considerados normais, apenas recentemente a importância dos sulfetos na dinâmica de metais passou a merecer atenção. A literatura recente mostra que estas espécies também podem desempenhar um papel importante no controle da especiação do cobre em águas de rios ${ }^{14,15,21}$.

Nestes ambientes, contudo, o papel dos sulfetos inorgânicos não tem recebido a mesma atenção que é dada à matéria orgânica, pois ainda existe a expectativa de que a concentração total de sulfetos seja muito reduzida em águas ricas em oxigênio ${ }^{15}$. Desta maneira, a importância dos sulfetos como agentes ligantes de metais tem sido associada apenas a ambientes anóxicos e igualmente à formação de sulfetos insolúveis. Entretanto, mesmo em águas de rios contendo quantidades significativas de oxigênio dissolvido, os sulfetos solúveis também devem ser avaliados em função de sua importância como agente de complexação de metais ${ }^{14,15,22,23}$. Rozan e Benoit ${ }^{14}$ observaram que os complexos de sulfeto com cobre presentes na coluna de água não se mostram reativos por um período de até 14 dias, mesmo sob condições óxicas. Isto ocorre, principalmente, devido às elevadas constantes de estabilidade, características dos complexos formados entre os compostos de sulfeto e metais como cobre, chumbo, zinco e ferro ${ }^{16}$. A estabilidade destes complexos e sua resistência à oxidação e dissociação são características relevantes para o controle da biodisponibilidade. De forma que o aumento da estabilidade cinética e termodinâmica, como resultado da formação de sulfetos, torna-se importante no controle da toxicidade frente à biota aquática sensível a metais ${ }^{21}$.

A concentração de sulfetos em águas naturais continentais depende, entre outros fatores, do nível de ocupação da bacia hidrográfica e das caraterísticas biogeoquímicas da região ${ }^{14}$. Rozan e colaboradores ${ }^{15}$ encontraram concentrações de sulfeto dissolvido consideradas elevadas, em rios de Connecticut e Maryland (EUA), com valores que alcançaram até $600 \mathrm{nmol} \mathrm{L}^{-1}$ em regiões mais impactadas, sendo mais de $90 \%$ desse sulfeto presente na forma de complexos de cobre, ferro e zinco. As quantidades encontradas são 
significativas, pois $20 \%$ do total de $\mathrm{Fe}$ e $\mathrm{Zn}$ e $45 \%$ do total de $\mathrm{Cu}$ presentes na fração dissolvida encontravam-se complexados com diferentes espécies de sulfeto. Nestes casos, a concentração total de sulfetos foi obtida pela somatória dos sulfetos de $\mathrm{Fe}, \mathrm{Cu}$ e Zn, além de complexos formados com $\mathrm{Pb}, \mathrm{Cd}, \mathrm{Hg}$ e $\mathrm{Ag}$, presentes em quantidades minoritárias, e espécies livres, tais como $\mathrm{HS}^{-}, \mathrm{S}_{\mathrm{x}}{ }^{2-}$ e $\mathrm{S}_{(\mathrm{s})}$.

Rozan e Benoit ${ }^{14}$ estudaram os principais fatores geoquímicos responsáveis pelo controle da especiação do cobre, em rios submetidos a diferentes níveis de impactação na Bacia do Rio Delaware (EUA), e observaram que a concentração total de complexos de sulfetos de cobre variava em função de aspectos naturais e antropogênicos. Concentrações inferiores de $\mathrm{CuS}$ foram encontradas em regiões menos urbanizadas, onde a presença destes complexos foi atribuída a processos naturais. Por outro lado, em rios que drenavam áreas densamente urbanizadas, foram detectados níveis superiores de complexos $\mathrm{CuS}$, além de concentrações de sulfetos de até $200 \mathrm{nmol} \mathrm{L}^{-1}$. Neste caso, o principal aspecto responsável pelo aumento nos níveis de $\mathrm{CuS}$ nos rios foi o descarte de efluentes gerados a partir de sistemas sépticos e de estações de tratamento de esgoto.

Acredita-se que as principais fontes de sulfeto para águas superficiais localizadas em regiões urbanas surjam a partir do descarte de esgotos sanitários tratados e não-tratados e de efluentes industriais provenientes, principalmente, de curtumes, indústrias de papel e celulose e refinarias de petróleo. Espécies de sulfeto também podem ocorrer, em águas naturais, a partir de fontes difusas relacionadas à drenagem superficial de águas. A redução de sulfatos, que ocorre em todos os tipos de solo, em maior ou menor intensidade, contribui para o aumento da concentração de sulfetos em águas naturais, uma vez que as espécies reduzidas de enxofre são solúveis em meio aquoso e podem ser carreadas para os cursos de água por meio do escoamento superficial de águas pluviais e de irrigação ${ }^{24}$. A utilização de fertilizantes e de defensivos agrícolas à base de enxofre também constituem fontes que colaboram de forma indireta para o aporte de sulfetos em águas naturais.

As formas reduzidas do enxofre desempenham um papel chave em processos biogeoquímicos, que podem provocar alterações na especiação de metais em águas naturais. Entretanto, os dados encontrados na literatura relacionam-se apenas a ambientes aquáticos localizados em países desenvolvidos, em condições diferentes daquelas dos rios situados no Brasil, principalmente no que se refere ao estado de preservação. Em função destes aspectos, o objetivo deste trabalho consistiu no estudo dos principais fatores responsáveis pelo controle da especiação do cobre em rios localizados na Região Metropolitana de Curitiba, sob diversos níveis de ocupação da bacia e de atividades antrópicas. Esta avaliação foi realizada a partir da determinação do cobre presente em diferentes formas físicas e químicas, considerando a influência de fatores geoquímicos e dos sulfetos solúveis.

\section{PARTE EXPERIMENTAL}

\section{Coleta e preparo de amostras}

Foram selecionados quatro pontos de coleta localizados nos rios Iraí, Belém, Iguaçu e Barigui, que compõem a Bacia do Alto Iguaçu, na Região Metropolitana de Curitiba (RMC). A Bacia Hidrográfica do Iguaçu é o maior complexo hídrico do estado do Paraná, com uma área de $55.024 \mathrm{~km}^{2}$. Suas nascentes localizam-se na frente meridional da Serra do Mar, nas proximidades de Curitiba e o rio estende-se por $1275 \mathrm{~km}$ até sua $\mathrm{foz}^{25}$. A região denominada Alto Iguaçu compreende o complexo hídrico situado na RMC, que tem uma população aproximada de 2,1 milhões de habitantes. A localização dos pontos de amostragem na RMC é mostrada na Figura 1.
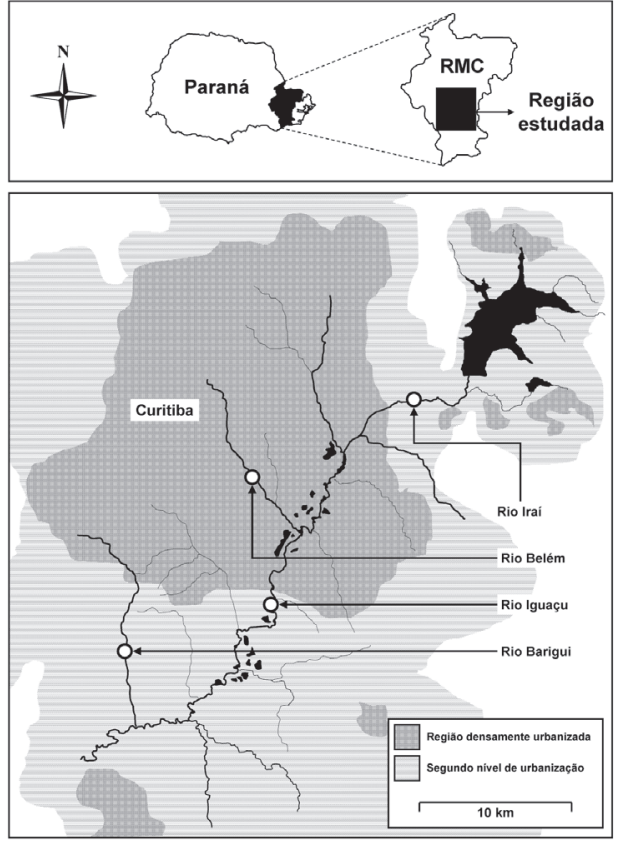

Figura 1. Localização dos pontos de amostragem na Bacia do Alto Iguaçu

$\mathrm{O}$ ponto de coleta no Rio Iraí localiza-se à montante da cidade de Curitiba e representa o ambiente menos impactado; o local de amostragem no Rio Belém situa-se na cidade de Curitiba em região densamente povoada; as amostras de águas do Rio Iguaçu foram coletadas à jusante da cidade, logo após a Estação de Tratamento de Esgoto - ETE Belém; e o quarto ponto de amostragem situa-se no Rio Barigui, que drena uma área predominantemente industrial ${ }^{25}$. Portanto, os locais de amostragem foram selecionados buscando-se uma variedade de níveis de ocupação da bacia hidrográfica, incluindo áreas da RMC com diferentes graus de urbanização, assim como regiões onde predomina a atividade industrial. Esta estratégia foi adotada para permitir a avaliação dos efeitos da ocupação da bacia tanto nos teores de sulfeto, quanto na especiação do cobre.

Amostras de águas superficiais dos rios foram coletadas e processadas segundo protocolos de técnicas limpas, de forma a minimizar processos de contaminação. Além disso, todos os procedimentos que envolveram as etapas de preservação, pré-tratamento e análise também foram realizados com os cuidados necessários para evitar contaminações, uma vez que nestas amostras o cobre ocorre em níveis traço ${ }^{26,27}$.

As amostras foram coletadas com auxílio de um balde de polietileno e, imediatamente, transferidas para frascos de polietileno pré-lavados e ambientados com a própria água de coleta. Para cada local de amostragem foram utilizados dois frascos de $1 \mathrm{~L}$ de capacidade. Em seguida, os frascos foram colocados individualmente em sacos plásticos e acondicionados em caixa de isopor com gelo. Este procedimento é importante para manter as amostras resfriadas até a chegada ao laboratório, como forma de minimizar a dessorção de metais do material particulado e perdas de sulfeto ${ }^{28}$.

No laboratório, as amostras foram imediatamente filtradas em um aparato de filtração, sob pressão reduzida, utilizando-se membranas de acetato de celulose com $0,45 \mu \mathrm{m}$ de porosidade (Schleicher \& Shuell), para obtenção da fração denominada dissolvida. Alíquotas reservadas para determinação da concentração total do cobre na fração dissolvida e na amostra in natura foram imediatamente acidificadas até $\mathrm{pH}<2$, com $\mathrm{HCl}$ purificado através de destilação isotérmica ${ }^{27}$. Em seguida, todas as amostras foram preservadas a 4 ${ }^{\circ} \mathrm{C}$, em refrigerador, até a realização das análises. 


\section{Determinação de CuS}

A identificação de $\mathrm{CuS}$ nas amostras foi realizada por voltametria de redissolução catódica com onda quadrada (VRCOQ) em alíquotas filtradas. Os parâmetros instrumentais empregados foram: eletrodeposição a $-0,1 \mathrm{~V}$ durante $180 \mathrm{~s}$ e varredura de potencial de $-0,1$ até $-1,4 \mathrm{~V}$ a $200 \mathrm{mV} \mathrm{s}^{-1}$, com freqüência de $100 \mathrm{~Hz}$ e amplitude de pulso de $50 \mathrm{mV}$.

Devido à possibilidade de perda de sulfetos por oxidação, as determinações foram realizadas até $3 \mathrm{~h}$ após a coleta. A identificação dos complexos $\mathrm{CuS}$ consistiu no monitoramento dos picos de redissolução do sulfeto em $-0,6 \mathrm{~V}(\mathrm{Ag} / \mathrm{AgCl})$ durante a titulação da amostra com $\mathrm{HNO}_{3} 0,01$ mol L-1 e determinação simultânea do pico de redissolução do cobre por voltametria de redissolução anódica com pulso diferenciado (VRAPD). O procedimento empregado para a identificação de $\mathrm{CuS}$ é apresentado na Figura 2.

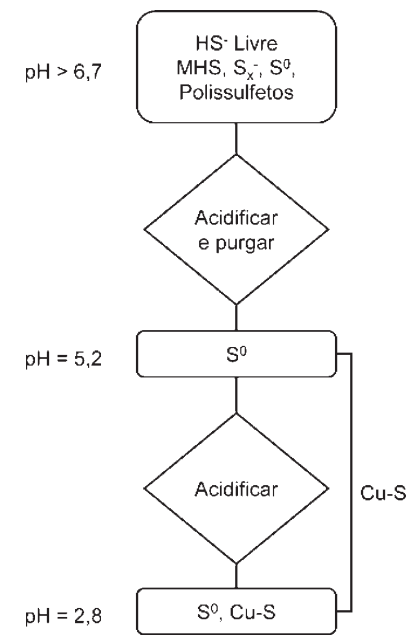

Figura 2. Representação esquemática do procedimento experimental empregado para identificação dos complexos de sulfeto com cobre. Adaptada das refs. 15 e 16

A partir de uma série de adições de ácido e purgas com $\mathrm{N}_{2}$ foi possível realizar uma determinação semi-quantitativa do sulfeto ligado ao cobre nas amostras de águas naturais, através do monitoramento do pico de redissolução do sulfeto em pH 5,0 e 2,8. Determinou-se também, de forma simultânea, a concentração de cobre por VRAPD, nos mesmos valores de $\mathrm{pH}$, como forma de se estimar a porcentagem do metal ligado ao sulfeto.

O monitoramento dos picos de redissolução foi realizado imediatamente após o término de cada etapa de adição de ácido ou purga. $\mathrm{O}$ $\mathrm{pH}$ das amostras foi determinado com um eletrodo de vidro combinado conectado a um potenciômetro Micronal B474. Os volumes de $\mathrm{HNO}_{3}$ necessários para o ajuste do $\mathrm{pH}$ durante a titulação voltamétrica foram obtidos em uma segunda alíquota da amostra preparada de forma idêntica àquela reservada para determinação voltamétrica do $\mathrm{CuS}$. A quantidade de ácido adicionada dependeu, entre outros fatores, do volume da amostra a ser acidificada e das características fisico-químicas de cada amostra, tais como alcalinidade, $\mathrm{pH}$ natural e conteúdo de carbono orgânico dissolvido. Durante as etapas de purga foi empregado $\mathrm{N}_{2}$ purificado em torres de lavagem contendo $\mathrm{V}$ (II) em meio áci-

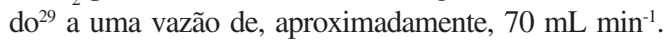

\section{Distribuição do cobre nos ambientes aquáticos}

Para determinação dos teores de cobre total dissolvido (CuTD) e cobre total recuperável (CuTR), alíquotas previamente acidificadas das frações dissolvida e in natura, respectivamente, foram digeridas empregando-se um reator fotoquímico ativado por microondas ${ }^{30}$. A determinação do cobre em ambas as alíquotas foi realizada por VRAPD, pelo método de adição de padrões, em um potenciostato EG\&G PAR M394 acoplado a um sistema de eletrodos SMDE EG\&G PAR 303A utilizando o modo gota pendente de mercúrio. Os parâmetros instrumentais para determinação de CuTR e CuTD foram: 10 min de purga com $\mathrm{N}_{2}$ e eletrodeposição a $-1,0 \mathrm{~V}$ durante $10 \mathrm{~min}$. Em seguida, foi feita uma varredura de potencial no sentido anódico, de -1,0 até $0,1 \mathrm{~V}$ a $8 \mathrm{mV} \mathrm{s}^{-1}$, com amplitude de pulso de $50 \mathrm{mV}$, sob força iônica do meio ajustada em 0,1 mol L-1 com $\mathrm{KNO}_{3}$ (Merck).

A concentração de cobre lábil também foi determinada por VRAPD, empregando-se os mesmos parâmetros instrumentais utilizados para determinação de CuTD e CuTR. Neste caso, uma alíquota da fração dissolvida e não acidificada foi titulada com uma solução $1,00 \mathrm{mg} \mathrm{L}^{-1}$ de cobre (Tritisol, Merck), sendo que após cada adição a amostra permanecia sob agitação constante durante $20 \mathrm{~min}^{31}$.

Os dados obtidos durante a titulação foram tratados empregando-se o modelo de linearização de van den Berg-Ruzic ${ }^{32,33}$, de acordo com a Equação 1:

$$
\frac{[\mathrm{Cu}]}{[\mathrm{CuL}]}=\frac{1}{K_{\mathrm{CuL}}[\mathrm{L}]}+\frac{1}{[\mathrm{~L}]}[\mathrm{Cu}]
$$

onde $[\mathrm{Cu}],[\mathrm{L}]$ e $[\mathrm{CuL}]$ representam as concentrações do cobre lábil, dos ligantes disponíveis à complexação e do complexo metal-ligante, respectivamente, enquanto que $K_{\mathrm{CuL}}$ indica a constante de estabilidade condicional para o complexo $\mathrm{CuL}$. A partir de um gráfico que relaciona $[\mathrm{Cu}] /[\mathrm{CuL}]$ em função de $[\mathrm{Cu}]$ são extraídos os valores de [L] e $K_{\mathrm{CuL}}$. O cobre lábil corresponde ao valor de $[\mathrm{Cu}]$ no primeiro ponto da curva de titulação ${ }^{34}$.

\section{Análises complementares}

As amostras dos rios também foram caracterizadas com relação a alguns parâmetros aquáticos. Alcalinidade total, teores de cloreto dissolvido e de sólidos suspensos totais (SST) foram determinados pelos métodos 2540D, 4500B e 2320B, respectivamente, descritos no "Standard Methods for the Examination of Water and Wastewater" ${ }^{35}$. Determinações de carbono orgânico dissolvido (COD) foram realizadas de acordo com o método SM5310B ${ }^{35} \mathrm{em}$ um analisador de carbono Shimadzu TOC-VCPH. Oxigênio dissolvido, condutividade, temperatura, $\mathrm{pH}$ e turbidez foram determinados in situ empregando-se um analisador de água portátil WQC 20A (TOA Eletronics).

\section{RESULTADOS E DISCUSSÃO}

\section{Caracterização dos rios estudados}

A caracterização dos corpos aquáticos foi realizada buscando conhecer os efeitos de ocupação da bacia sobre a qualidade das águas e, também, para subsidiar o estudo da especiação do cobre. A Tabela 1 mostra os resultados obtidos para determinação de alguns parâmetros aquáticos em amostras representativas dos rios estudados.

A partir dos dados mostrados na Tabela 1 observa-se que o $\mathrm{pH}$ apresentou valores entre 6,5 e 7,6, que são típicos para os rios da região ${ }^{25}$. Os níveis de alcalinidade foram superiores para os rios mais afetados por atividades antrópicas, com valores que variaram de 116,3 a 133,0 mg $\mathrm{CaCO}_{3} \mathrm{~L}^{-1}$. Por outro lado, no Rio Iraí a alcalinidade total foi cerca de quatro vezes inferior àquela dos demais rios. Níveis mais elevados para este parâmetro podem ser atribuídos a inúmeras fontes, dentre elas o descarte de águas resi- 
Tabela 1. Parâmetros aquáticos determinados para amostras representativas dos rios Iraí, Belém, Iguaçu e Barigui

\begin{tabular}{lcccccc}
\hline Rios & $\mathrm{pH}$ & $\begin{array}{c}\text { Alcalinidade } \\
\mathrm{mg} \mathrm{CaCO}_{3} \mathrm{~L}^{-1}\end{array}$ & OD & $\begin{array}{c}\text { Cloreto } \\
\mathrm{mg} \mathrm{L}^{-1}\end{array}$ & $\begin{array}{c}\mathrm{COD} \\
\text { SST }\end{array}$ \\
\hline Iraí & 6,5 & 33,30 & 6,7 & 2,47 & 6,80 & 8,6 \\
Belém & 7,4 & 116,3 & 1,1 & 32,7 & 11,7 & 30,8 \\
Iguaçu & 7,0 & 123,7 & 0,2 & 32,7 & 8,60 & 46,8 \\
Barigui & 7,6 & 133,0 & 1,2 & 21,8 & 7,80 & 6,3 \\
\hline
\end{tabular}

OD: oxigênio dissolvido; COD: carbono orgânico dissolvido; SST: sólidos suspensos totais

duais tratadas e/ou não tratadas para corpos aquáticos ${ }^{36,37}$. Além disso, valores mais elevados de alcalinidade também decorrem da decomposição de nutrientes e substratos orgânicos, sob condições anaeróbias $^{38}$. De fato, os rios Iguaçu, Belém e Barigui apresentaram níveis mais baixos de oxigênio dissolvido, quando comparados a valores obtidos para amostras Rio Iraí, conforme mostrado na Tabela 1. O desenvolvimento de ambientes anóxidos favorece a proliferação de microorganismos anaeróbios redutores de sulfato, fazendo com que o corpo aquático apresente concentrações mais elevadas de espécies como mercaptanas e sulfetos ${ }^{36}$.

Além de contribuir para alterações nos níveis de alcalinidade e OD em águas naturais, pontos próximos a descargas de esgoto apresentam taxas mais elevadas de produção primária, que contribuem para o aumento nos níveis de COD nestes ambientes ${ }^{39}$. Na Tabela 1, podese observar que a concentração de COD foi maior para o Rio Belém, seguido dos rios Iguaçu, Barigui e Iraí. Os valores superiores de COD nas amostras coletadas em pontos localizados na cidade de Curitiba e à sua jusante são indicativos de contribuições antropogênicas, provavelmente associadas ao descarte de esgoto. As concentrações de SST também foram superiores para os rios Belém e Iguaçu, em comparação aos outros dois corpos aquáticos. Alguns aspectos podem ser responsáveis pelo aumento dos níveis de sólidos nos rios que sofrem, de maneira mais acentuada, os efeitos dos processos de urbanização ${ }^{40}$.

Sodré e colaboradores ${ }^{41}$ evidenciaram que o aporte de sólidos em rios da RMC ocorre tanto a partir de contribuições difusas, quanto por meio de fontes pontuais. No caso do Rio Barigui, cujo ponto de coleta encontra-se em área industrial, a concentração de SST foi, entretanto, compatível a ambientes menos impactados ${ }^{16}$. Considerando que neste rio foram observados valores elevados para os demais parâmetros indicativos de contribuições antropogênicas, acredita-se que as fontes de poluição na região industrial diferem daquelas dos pontos de amostragem localizados nos rios sob maior influência dos descartes de efluentes de origem doméstica.

Portanto, os parâmetros aquáticos analisados indicam uma forte influência do nível de ocupação da bacia nas características dos rios estudados. O Rio Iraí mostrou-se menos impactado pelas atividades antrópicas, enquanto que os rios Belém e Iguaçu apresentaram um elevado nível de deterioração provocado, principalmente, pelo descarte de esgoto doméstico a partir da cidade de Curitiba. De fato, a questão de maior gravidade no quadro ambiental dos rios da RMC é a poluição causada pelo descarte clandestino de esgoto doméstico ${ }^{42}$. Por outro lado, para o Rio Barigui, cujo ponto de coleta está localizado na área industrial, foram observadas características distintas no que diz respeito à impactação, sugerindo influência das atividades desenvolvidas na região da bacia sobre este corpo aquático.

\section{Distribuição do cobre}

Os resultados obtidos para distribuição do cobre em amostras representativas dos quatro rios são mostrados na Tabela 2, onde aparecem os valores de $\mathrm{CuTR}, \mathrm{CuTD}, \mathrm{Cu}$ lábil e $\mathrm{Cu}-\mathrm{P}$, que correspondem à concentração de cobre no material particulado em suspensão normalizado em função do teor de SST. Pode-se observar que, para os quatro rios estudados, as concentrações de CuTR variaram de 2,35 a 31,4 $\mu \mathrm{g}$ $\mathrm{L}^{-1}$, evidenciando que existem diferenças com relação ao teor de cobre em função dos níveis de ocupação da bacia. Os rios Belém e Iguaçu, mais impactados em função do aporte de efluentes gerados a partir da região mais densamente urbanizada, apresentaram as maiores concentrações de CuTR. O aporte de esgoto bruto e tratado constitui a principal fonte de cobre em rios localizados na cidade de Curitiba ${ }^{41}$.

A concentração de CuTR no Rio Iraí, de 7,64 $\mu \mathrm{g} \mathrm{L}^{-1}$, mostra-se compatível com os níveis de cobre determinados em ambientes relativamente mais preservados da $\mathrm{RMC}^{41}$. É ainda interessante observar que o teor de CuTR no Rio Barigui foi o menor dentre os quatro rios, apesar desse ponto de coleta estar localizado em região predominantemente industrial.

As concentrações de CuTD variaram de 9,23 a 1,40 $\mu \mathrm{g} \mathrm{L}^{-1}$ para os quatro rios, sendo que o Rio Belém também apresentou os maiores teores de cobre na fração dissolvida, seguido dos rios Iraí, Iguaçu e Barigui. Concentrações mais elevadas de CuTD implicam em um maior tempo de residência do metal na coluna de água, que pode ser mais facilmente disponibilizado.

Na Tabela 2 pode-se observar que os teores de $\mathrm{Cu}-\mathrm{P}$ foram mais elevados para as amostras coletadas nos rios Belém e Iguaçu. Dependendo das características das partículas em suspensão, espécies metálicas podem permanecer preferencialmente associadas a este materi$\mathrm{al}^{43,44}$. Freqüentemente, o material particulado encontra-se recoberto por matéria orgânica de ocorrência natural, resultando em capacidades elevadas de adsorção de metais em suas superfícies ${ }^{45,46}$. Neste caso, este material exerce um importante papel na disponibilidade do metal.

Tabela 2. Resultados obtidos para especiação do cobre em amostras representativas de rios da RMC

\begin{tabular}{lcccc}
\hline Rios & CuTR & $\begin{array}{c}\text { CuTD } \\
\mu \text { L L}^{-1}\end{array}$ & Cu lábil & $\begin{array}{c}\mathrm{Cu}-\mathrm{P} \\
\mathrm{mg} \mathrm{kg}^{-1}\end{array}$ \\
\hline Iraí & 7,64 & 6,55 & 0,45 & 126,7 \\
Belém & 31,38 & 9,23 & 0,14 & 719,2 \\
Iguaçu & 13,42 & 2,97 & 1,67 & 223,3 \\
Barigui & 2,35 & 1,40 & $<0,01$ & 150,8 \\
\hline
\end{tabular}

CuTR: cobre total recuperável; CuTD: cobre total dissolvido; $\mathrm{Cu}-$ $\mathrm{P}$ : cobre no material particulado em suspensão, $\mathrm{Cu}-\mathrm{P}=(\mathrm{CuTR}-$ $\mathrm{CuTD}) /(\mathrm{SST} \times 1000)$.

Muitos trabalhos publicados na década de 70 mostravam que a disponibilidade do cobre para a biota era governada apenas pela presença do íon livre em solução ${ }^{47-51}$. Mais recentemente, entretanto, foi evidenciado que a concentração lábil, que compreende além do íon livre as formas fracamente ligadas, é a que está mais intimamente vinculada à biodisponibilidade do metal, e conseqüentemente, à sua toxicidade ${ }^{31,52}$. Na Tabela 2, observa-se que a concentração de $\mathrm{Cu}$ lábil variou de 1,67 até valores inferiores a $0,01 \mu \mathrm{g} \mathrm{L}^{-1}$ para os quatro rios avaliados. A amostra coletada no Rio Belém apresentou a menor concentração de $\mathrm{Cu}$ lábil devido, provavelmente, às concentrações elevadas de COD e SST encontradas neste rio (Tabela 1), que podem contribuir com sítios de complexação/adsorção de metais ${ }^{6,12}$. Por outro lado, a amostra do Rio Iguaçu, apesar de também conter níveis elevados de SST e COD, apresentou a maior concentração de Cu lábil dentre os quatro rios avaliados. Porém, considerando o conteúdo de CuTR nesta amostra, verifica-se que em termos relativos, a fração lábil contribuiu com apenas cerca de $12 \%$ do metal presente na coluna de água.

A avaliação da distribuição do cobre nos rios também foi realizada empregando-se dados referentes à especiação do metal, conforme mostra a Figura 3, para os quatro rios avaliados. 
No gráfico de barras da Figura 3, CuL representa a fração de cobre complexado na fração dissolvida e $\mathrm{Cu}_{\mathrm{p}}$ a porcentagem de cobre associado ao material particulado. A somatória entre $\mathrm{CuL}$, $\mathrm{Cu}_{\mathrm{p}}$ e $\mathrm{Cu}$ lábil é igual ao teor total de cobre (CuTR) nas amostras. Pode-se observar que existem diferenças significativas com relação à distribuição do cobre na coluna de água para os corpos aquáticos, nos pontos avaliados. No Rio Iguaçu, cerca de $80 \%$ do CuTR encontra-se associado ao material particulado em suspensão, seguido dos rios Belém (70\%), Barigui (40\%) e Iraí (15\%). As maiores porcentagens de $\mathrm{Cu}_{\mathrm{p}}$ foram obs. para aquelas amostras coletadas em ambientes onde se evidencia maior expansão urbana sobre a bacia, enquanto que no ambiente menos impactado o cobre permaneceu, preferencialmente, na fração dissolvida. Além de apontar diferenças com relação à distribuição do metal em função do nível de ocupação e atividade na região da bacia, estes resultados sugerem que existe uma transferência do cobre da fração dissolvida para o material particulado, conforme a coluna de água se torna mais impactada pelas atividades antrópicas.

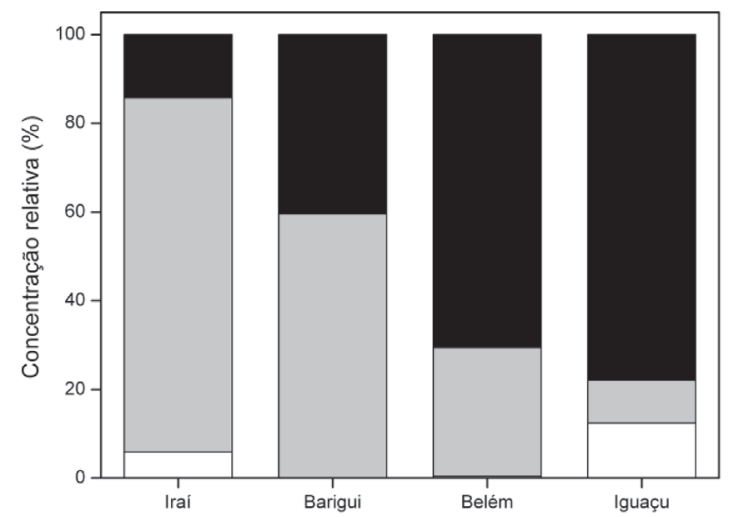

Figura 3. Distribuição relativa do cobre entre as frações particulada $\left(C u_{p}\right.$ em preto), complexada solúvel (CuL em cinza) e lábil (em branco) nos quatro rios estudados

Em águas naturais, o material particulado pode conter inúmeras substâncias e materiais capazes de complexar ou adsorver metais, tais como argilas, oxi-hidróxidos de ferro e alumínio ${ }^{53-56}$, partículas orgânicas bióticas ou abióticas ${ }^{5,57,58}$, além de sólidos inorgânicos recobertos por matéria orgânica ${ }^{45,46}$.

Acredita-se que a maior porcentagem de cobre associado ao material particulado nos rios Belém e Iguaçu possa ser conseqüência do recobrimento deste material com matéria orgânica. Neste caso, a matéria orgânica particulada pode apresentar uma elevada reatividade e conferir sítios disponíveis à complexação do cobre, que competem com os agentes complexantes presentes na fração dissolvida. Peart e Walling ${ }^{59}$ avaliaram as características do material particulado na Bacia do Rio Devon, na Inglaterra, e evidenciaram que o enriquecimento das partículas em suspensão com matéria orgânica foi o principal fator responsável pela remoção de vários elementos da coluna de água.

A avaliação do grau de associação de metais junto ao material particulado e a presença do mesmo na fração dissolvida também foi realizada em termos do coeficiente de partição, $K_{\mathrm{D}}$, que é representado pela razão entre $\mathrm{Cu}-\mathrm{P}$ e $\mathrm{CuTD}$. A partição de uma espécie metálica em águas naturais exerce um papel importante no controle da especiação, uma vez que o conhecimento das condições que controlam a partição permite avaliar até que ponto uma espécie metálica pode ser transferida para formas biodisponíveis ${ }^{52}$.

A Figura 4 mostra a variação dos valores de $\log K_{\mathrm{D}}$ em função do $\mathrm{pH}$ para as amostras representativas dos quatro rios avaliados. Pode ser observado que os coeficientes de partição, em termos logarítmicos, variaram na faixa de 4,3 a 5,0 para os rios avaliados, mesmo em uma faixa de variação de $\mathrm{pH}$ de apenas 1,2 unidades. $\mathrm{Lu}$ e Allen ${ }^{60}$ mostraram que, mesmo nesta faixa de $\mathrm{pH}$, os valores de $K_{\mathrm{D}}$ variaram significativamente para diferentes valores de CuTR e SST. Na Figura 4, observa-se também que os valores de $\log K_{\mathrm{D}}$ são mais elevados em função do aumento do $\mathrm{pH}$, mostrando que o cobre tende a permanecer na fração dissolvida nas amostras que apresentaram os valores mais baixos de $\mathrm{pH}$. Gundersen e Steinnes ${ }^{61}$ avaliaram a influência do $\mathrm{pH}$ na partição de metais em águas naturais e mostraram que em ambientes mais ácidos, com $\mathrm{pH}$ variando entre 3 e 6 , menos de $10 \%$ do total do cobre encontrava-se associado ao material particulado. O restante, cerca de $90 \%$, encontrava-se presente na fração dissolvida. Neste caso, o aumento na concentração de íons $\mathrm{H}^{+}$em solução pode deslocar os íons $\mathrm{Cu}^{2+}$ complexados em sítios disponíveis tanto na fração dissolvida ${ }^{61}$, quanto no material particulado ${ }^{45}$. Uma vez que nos quatro rios avaliados a concentração de cobre encontra-se na faixa de $\mu \mathrm{g} \mathrm{L}^{-1}$, o deslocamento do $\mathrm{pH}$ para valores próximos de 6 , por ex., resulta em níveis de $\mathrm{H}^{+}$superiores aos do metal em até três ordens de grandeza. Neste trabalho, o Rio Iraí apresentou o menor valor de $\mathrm{pH}$ em comparação aos demais rios e também a maior porcentagem de cobre na fração dissolvida. Deste modo, acredita-se que a competição entre íons $\mathrm{H}^{+}$ e $\mathrm{Cu}^{2+}$ também exerceu um papel importante na especiação do metal na coluna de água.

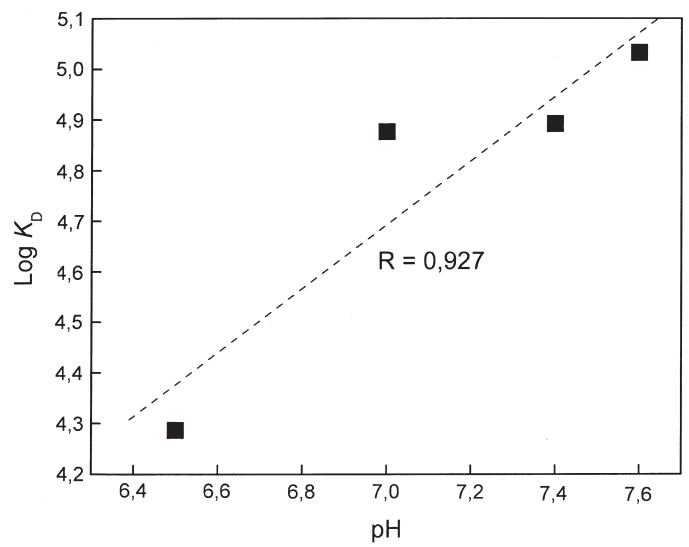

Figura 4. Coeficientes de partição do cobre $\left(\mathrm{K}_{D}\right)$ em função dos valores de pH para amostras representativas dos rios Iraí, Belém, Iguaçu e Barigui

\section{Complexação de cobre por sulfetos solúveis}

Tendo por base o pH de dissociação foi possível, através de medidas eletroquímicas, distinguir sulfetos liberados de complexos metálicos presentes nas amostras dos corpos aquáticos estudados, durante o procedimento de titulação ácida acompanhado de purgas com $\mathrm{N}_{2}$. O protocolo empregado neste trabalho prevê a determinação da corrente de redissolução do sulfeto empregando-se VRCOQ, com eletrodo de mercúrio de gota pendente.

A determinação do sulfeto por VRCOQ originou voltamogramas como os mostrados na Figura 5, onde podem ser observados os picos relativos às espécies eletroativas de sulfeto na amostra, em diferentes valores de $\mathrm{pH}$, durante o procedimento de titulação ácida.

Nas determinações, o pico inicial de sulfeto em $\mathrm{pH}$ natural da amostra representa todas as espécies eletroativas de enxofre presentes, tais como $\mathrm{S}^{0}, \mathrm{HS}^{-}, \mathrm{S}^{2-}, \mathrm{S}_{\mathrm{x}}^{-2}$, que contribuem para o pico observado em potencial aproximadamente igual a -0,6V. Após acidificação para pH 6,7 os sulfetos de $\mathrm{Co}, \mathrm{Fe}, \mathrm{Mn}$, Ni sofreram dissociação e o íon sulfeto liberado formou $\mathrm{H}_{2} \mathrm{~S}$ que foi eliminado pela purga. O pico restante indica $\mathrm{S}_{\mathrm{x}}^{2-}$ livre e $\mathrm{S}^{0}$. Observou-se uma 


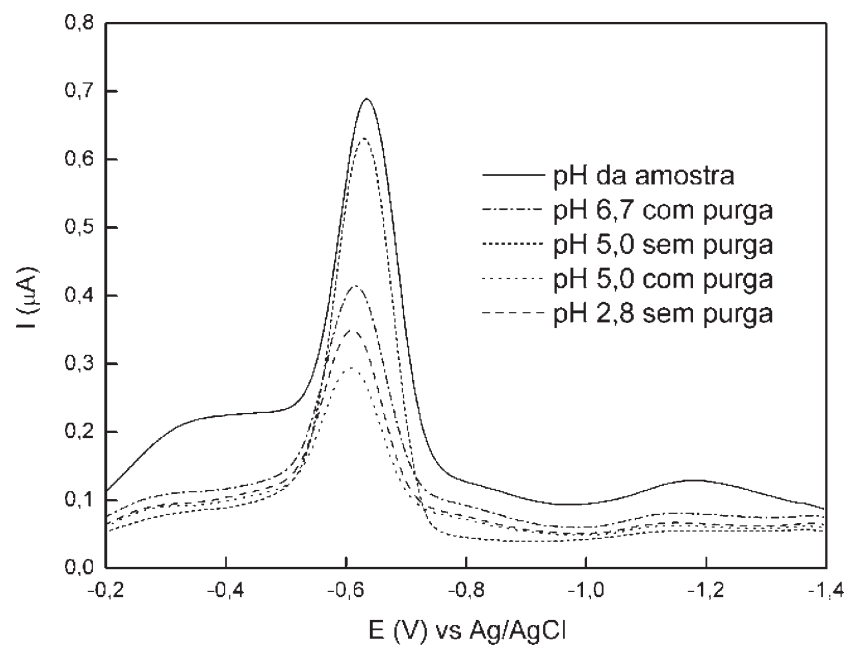

Figura 5. Voltamogramas obtidos para determinação de sulfetos em amostras de águas naturais utilizando VRCOQ

redução na intensidade do pico, em decorrência da purga e, portanto, da eliminação do sulfeto eletroativo produzido pela dissociação.

$\mathrm{Na}$ etapa seguinte, quando se acidificou para pH 5,0 sem purga, o sulfeto medido representou $\mathrm{S}_{\mathrm{x}}{ }_{\mathrm{x}}^{2-}$ livre e $\mathrm{S}^{0}$ remanescentes da etapa anterior, mais o sulfeto liberado pela dissociação de sulfetos de $\mathrm{Zn}$, de $\mathrm{Pb}$ e de Fe solúvel.

Depois de realizar a medida em $\mathrm{pH} 5,0$ purgou-se por $300 \mathrm{~s} \mathrm{e}$ procedeu-se a nova leitura. Nesse valor de $\mathrm{pH}$, após a purga, apenas $\mathrm{S}^{0}$ foi medido, pois os polissulfetos também foram eliminados como $\mathrm{H}_{2} \mathrm{~S}$ durante a etapa de purga. A espécie $\mathrm{S}^{0}$ é restante da etapa anterior ou resultante da dissociação de polissulfetos, que ocorre em $\mathrm{pH}<6,5$ para $\mathrm{S}_{4}{ }^{2-}$ e $\mathrm{pH}<6,1$ para $\mathrm{S}_{5}{ }_{5}^{2-}$. Esse foi o pico de menor intensidade observado nas medidas.

O próximo passo foi a acidificação para $\mathrm{pH}$ 2,8 sem purga. Nesse valor de $\mathrm{pH}$, o sulfeto de $\mathrm{Cu}$ presente na amostra sofreu dissociação e foi medido juntamente com o $\mathrm{S}^{0}$ restante da etapa anterior. Houve um pequeno aumento na intensidade do pico e a diferença dos valores de corrente entre $\mathrm{pH}$ 5,0 e 2,8 permitiu identificar o sulfeto ligado ao cobre.

Os procedimentos experimentais incluíram, paralelamente, a determinação do cobre dissolvido através de VRAPD, durante a titulação ácida para sulfeto.

Nesta determinação, a concentração de cobre ligado ao sulfeto foi calculada considerando-se a concentração deste metal em $\mathrm{pH}$ 5,0 e depois da acidificação em $\mathrm{pH}$ 2,8. Este procedimento, entretanto, permitiu apenas estimar os valores em termos relativos para os pontos de amostragem, pois algumas limitações foram constatadas. A acidificação do meio interferiu no sinal analítico do cobre provocando um aumento na intensidade da corrente, que não significou aumento da concentração de $\mathrm{Cu}^{2+}$, e ainda, a dissociação do cobre em valores de $\mathrm{pH}$ inferiores a 5,0 não pode ser atribuída exclusivamente a complexos $\mathrm{CuS}$, mas a todas as formas complexadas do cobre presentes na amostra. Apesar disso, comparando-se as concentrações de cobre em pH 5,0 e 2,8, determinadas nas mesmas condições analíticas, foi possível verificar diferenças no comportamento e nas interações que ocorrem com o cobre nestes sistemas.

De maneira geral, nas amostras coletadas nos ambientes mais impactados, como os rios Belém e Iguaçu, os sulfetos solúveis exerceram um papel determinante para o controle da especiação do cobre na fração dissolvida. Os resultados obtidos demonstram ainda a importância da contribuição dos sulfetos solúveis para a compreensão dos aspectos que governam a especiação de metais em águas naturais.

A porcentagem de $\mathrm{CuS}$ determinado na fração dissolvida, para amostras coletadas na RMC, em função da concentração de OD nestes ambientes está representada na Figura 6. A partir dos resultados, pode-se observar que a concentração de $\mathrm{CuS}$ diminuiu em função do aumento nos níveis de OD.

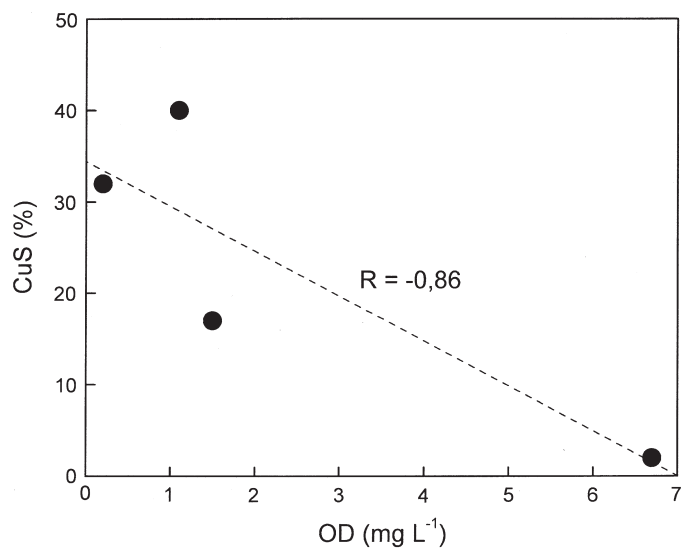

Figura 6. Variação da porcentagem de CuS na fração dissolvida em função da concentração de oxigênio dissolvido em amostras coletadas em rios da RMC

Estes resultados permitem evidenciar que o desenvolvimento de ambientes anóxidos, principalmente em função do aporte de esgotos nos rios localizados em regiões urbanas, favorece a geração de espécies reduzidas de enxofre nestas águas que, por sua vez, são capazes de complexar metais na coluna de água.

Entretanto, mesmo em ambientes menos impactados pela atividade antrópica, onde as concentrações de OD são mais elevadas ( $>5$ $\mathrm{mg} \mathrm{L}^{-1}$ ), os sulfetos solúveis também contribuíram para a complexação do cobre na fração dissolvida. No caso do Rio Iraí, por ex., cerca de $2 \%$ do teor de CuTD foi encontrado na forma de CuS devido, provavelmente, à ocorrência de sulfetos oriundos de processos naturais. Rozan e Benoit ${ }^{14}$ estudaram os principais fatores que controlam a especiação do cobre em rios localizados na Bacia do Rio Delaware e observaram que, em rios localizados em regiões mais desenvolvidas, as concentrações de $\mathrm{CuS}$ foram cerca de quatro vezes maiores que em rios mais preservados. Na Figura 6, pode-se observar ainda que, para os pontos localizados na RMC, os níveis de $\mathrm{CuS}$ foram cerca de 20 vezes maiores nos ambientes mais impactados. Esta maior diferença entre os ambientes estudados, quando comparada aos resultados mostrados por Rozan e Benoit ${ }^{14}$, evidencia que o aporte de esgotos constitui um dos principais aspectos responsáveis pela presença de complexos de sulfetos com o cobre em rios localizados em regiões densamente urbanizadas desta bacia.

\section{CONCLUSÕES}

Os resultados obtidos neste trabalho permitiram elucidar importantes aspectos que governam a especiação do cobre em rios localizados em uma região urbana altamente influenciada pelas atividades antrópicas. A partir da determinação de parâmetros aquáticos, foi possível diferenciar os rios estudados com relação aos seus níveis de impactação, verificando-se que as amostras coletadas em pontos à jusante e na cidade de Curitiba apresentaram-se afetadas, principalmente, pelo aporte de efluentes decorrentes da urbanização.

Os resultados obtidos para a distribuição do cobre nos rios estudados revelaram que houve maior transferência do cobre da fração dissolvida para o material particulado em função do grau de deterioração das águas dos rios. Neste caso, o pH e a presença de matéria orgânica particulada exerceram um papel importante para a distribuição do cobre na coluna de água. Além disso, foi evidenciado 
que os sulfetos solúveis também são importantes para a compreensão dos aspectos que controlam a especiação do cobre em águas naturais. A influência dos sulfetos foi maior em ambientes onde existe maior expansão urbana sobre a bacia e que apresentaram os menores valores de OD. Entretanto, foi mostrado que estes ligantes podem contribuir para a complexação de cobre mesmo em sistemas aquáticos ricos em oxigênio.

\section{REFERÊNCIAS}

1. Xue, H. B.; Sunda, W. G.; Environ. Sci. Technol. 1997, 31, 1902.

2. Tessier, A.; Fortin, D.; Belzile, N.; Devitre, R. R.; Leppard. G. G.; Geochim. Cosmochim. Acta 1996, 60, 387.

3. Sondi, I.; Pravdic, V.; Croat. Chem. Acta 1998, 71, 1061.

4. Stumm, W.; Morgan, J. J.; Aquatic chemistry, $3^{\text {rd }}$ ed., Wiley: New York, 1996.

5. Warren, L. A.; Haack, E. A.; Earth-Sci. Rev. 2001, 54, 261.

6. Campbell, P. C. G. Em Metal speciation and bioavailability in aquatic systems; Tessier, A.; Turner, D., eds.; John Wiley and Sons: Chichester, 1995, cap. 2.

7. Florence, T. M.; Talanta 1982, 29, 345.

8. Cabaniss, S. E.; Shuman, M. S.; Geochim. Cosmochim. Acta 1988, 52, 185.

9. Gerringa, L. J. A.; Hummel, H.; Moerdijk-Poortvliet, T. C. W.; J. Sea Res. 1998, 40, 193

10. Botelho, C. M. S.; Boaventura, R. A. R.; Gonçalves, M. D. S. S.; Electroanalysis 2002, 14, 1713.

11. Vasconcelos, M. T. S. D.; Leal, M. F. C.; van den Berg, C. M. G.; Mar. Chem. 2002, 77, 187.

12. McGeer, J. C.; Szebedinszky, D. C.; McDonald, G.; Wood, C. M.; Comp. Biochem. Physiol., Part C: Toxicol. Pharmacol. 2002, 133, 147.

13. Takács, M.; Alberts, J. J.; Egeberg, P. K.; Environ. Int. 1999, 25, 315.

14. Rozan, T. F.; Benoit, G.; Geochim. Cosmochim. Acta 1999, 63, 3311.

15. Rozan, T. F.; Lassman, M. E.; Ridge, D. P.; Luther III, G. W.; Nature 2000, $406,879$.

16. Luther III, G. W.; Theberge, S. M.; Rickard, D. T.; Geochim. Cosmochim. Acta 1999, 63, 3159.

17. Cutter, G. A.; Oatts, T. J.; Anal. Chem. 1987, 59, 717.

18. Cutter, G. A.; Velinsky, D. J.; Mar. Chem. 1988, 23, 311.

19. Luther III, G. W.; Tsamakis, E.; Mar. Chem. 1989, 27, 165

20. Zhang, J.; Millero, F. J.; Anal. Chim. Acta 1994, 284, 497.

21. Bowles, K. C.; Ernste, M. J.; Kramer, J. R.; Anal. Chim. Acta 2003, 477, 113.

22. Bianchini, A.; Bowles, K. C.; Comp. Biochem. Physiol., Part C: Toxicol. Pharmacol. 2002, 133, 51.

23. Allen, H. E.; Sci.Total Environ. 1993, Suppl 1, 23.

24. Giblin, A. E.; Wieder, R. K. Em Sulphur Cycling on the Continents: Wetlands, Terrestrial Ecosystems and Associated Water Bodies; Howarth, R. W.; Stewart, J. W. B.; Ivanov, M. V. eds.; John Wiley and Sons: Chichester, 1992, cap. 5.

25. Superintendência de Desenvolvimento de Recursos Hídricos e Saneamento Ambiental; Qualidade das águas interiores do Estado do Paraná: 19871995. Curitiba, 1997.

26. Allen, H. E.; Hum. Ecol. Risk Assess. 2000, 6, 989

27. Campos, M. L. A. M.; Bendo, A.; Viel, F. C.; Quim. Nova 2002, 25, 808.

29. Luther III, G. W.; Rickard, D.; Theberge, S. M.; Olroyd, A.; Environ. Sci. Technol. 1996, 30, 671.
29. Harris, D. C.; Quantitative Chemical Analysis, $6^{\text {th }}$ ed., W. H. Freeman and Company: New York, 2003.

30. Sodré, F. F.; Peralta-Zamora, P. G.; Grassi, M. T.; Quim. Nova 2004, 27, 695.

31. Mylon, S.; Twining, B. S.; Fisher, N. S.; Benoit, G.; Environ. Sci. Technol. 2003, 37, 1261 .

32. van den Berg, C. M. G.; Kramer, J. R.; Anal. Chim. Acta 1979, 106, 113.

33. Ruzic, I.; Anal. Chim. Acta 1982, 140, 99.

34. Donat, J. R.; Lao, K. A.; Bruland, K. W.; Anal. Chim. Acta 1994, 284, 547.

35. American Public Health Association, American Water Works Association, Water Environment Federation; Standard Methods for the Examination of Water and Wastewater, $19^{\text {th }}$ ed., WEF: Washington, 1995.

36. Rozan, T. F.; Benoit, G.; Luther III, G. W.; Environ. Sci. Technol. 1999 33, 3021.

37. Tchobanoglous, G.; Burton, F. L.; Wastewater Engineering: Treatment, Disposal and Reuse, $3^{\text {rd }}$ ed., McGraw-Hill Inc.: New York, 1991.

38. Verbanck, M.; Vanderborght, J. P.; Wollast, R.; Res. J. Water Pollut. Control Fed. 1994, 61, 1722.

39. Abril, G.; Frankignoulle, M.; Water Res. 2001, 35, 844.

40. Prestes, E. C.; dos Anjos, V. E.; Sodré, F. F.; Grassi, M. T.; J. Braz. Chem. Soc. 2006, 17, 53.

41. Sodré, F. F.; dos Anjos, V. E.; Prestes, E. C.; Grassi, M. T.; J. Environ. Monit. 2005, 7, 581

42. Coordenação da Região Metropolitana de Curitiba; Relatório Ambiental da Região Metropolitana de Curitiba, Governo do Estado do Paraná: Curitiba, 1997.

43. Stiff, M. J.; Water Res. 1971, 5, 585 .

44. Windom, H. L.; Byrd, T.; Smith, R. G.; Huan, F.; Environ. Sci. Technol. 1991, 25, 1137.

45. Grassi, M. T.; Shi, B.; Allen, H. E.; J. Braz. Chem. Soc. 2000, 11, 516.

46. Shi, B.; Allen, H. E.; Grassi, M. T.; Ma, H.; Water Res. 1998, 32, 3756.

47. Brown, V. M.; Shaw, T. L.; Shurben, D. G.; Water Res. 1974, 8, 797.

48. Chakoumakos, C.; Russo, R. C.; Thurston, R.; Environ. Sci. Technol. 1979, $13,213$.

49. Howarth, R. S.; Sprague, J. B.; Water Res. 1978, 12, 455.

50. Pagenkopf, G. K.; Russo, R. C.; Thurston, R. V.; J. Fish. Res. Bd. Can. 1974, 31, 462 .

51. Sunda, W.; Guillard, R. R. L.; J. Mar. Res. 1976, 34, 511.

52. Meylan, S.; Odzak, N.; Behra, R.; Sigg, L.; Anal. Chim. Acta 2004, 510, 91.

53. Harsh, J. B.; Doner, H. E.; Soil Sci. Soc. Am. J. 1984, 48, 1034.

54. Sigg, L. Em Chemical and Biological Regulation of Aquatic Systems; Buffle, J.; De Vitre, R. R., eds.; CRC Press: Boca Raton, 1994, cap. 5.

55. Martinez, C. E.; McBride, M. B.; Environ. Sci. Technol. 1998, 32, 743.

56. Honeyman, B. D.; Santschi, P. H.; Environ. Sci. Technol. 1988, 22, 862.

57. Bruland, K. W.; Donat, J. R.; Hutchins, D. A.; Limnol. Oceanogr. 1991, 36, 1555.

58. Sigg, L. Em Metals in surface waters; Allen, H. E.; Garrison, A. W.; Luther III, G W., eds.; Ann Arbor Press: Chelsea, 1998, cap. 11.

59. Peart. M. R.; Walling, D. E. Em Drainage basin sediment delivery; Hadley R. F., ed.; IAHS Press: Wallingford, 1986, cap. 4.

60. Lu, Y.; Allen, H. E.; Sci. Total Environ. 2001, 277, 119.

61. Gundersen, P.; Steinnes, E.; Water. Res. 2003, 37, 307. 